\title{
ARTIGO TÉCNICO \\ CUSTO DE BOMBAS CENTRÍFUGAS FUNCIONANDO COMO TURBINAS EM MICROCENTRAIS HIDRELÉTRICAS
}

\author{
CARLOS R. BALARIM ${ }^{1}$, LUIZ A. TARGA ${ }^{2}$, JORIM S. VIRGENS FILHO ${ }^{3}$, \\ ALCEU G. ANDRADE FILHO ${ }^{4}$, GIOVANA K. WIECHETECK ${ }^{5}$
}

\begin{abstract}
RESUMO: A implantação de microcentrais hidrelétricas é uma das alternativas para suprir com energia comunidades pequenas e isoladas, situadas normalmente na área rural. $\mathrm{O}$ aproveitamento de potenciais hidráulicos de pequeno porte é uma alternativa cada vez mais viável devido não só à falta de recursos financeiros para os grandes empreendimentos, mas também pelo imenso potencial de geração em centrais de pequeno porte, que pouco tem sido aproveitado. O objetivo deste trabalho foi de apresentar uma metodologia simples de engenharia para estimar o custo das Bombas Funcionando como Turbinas (BFTs) utilizadas em microcentrais hidrelétricas, que possam ser usadas em estudos preliminares de novos aproveitamentos hidrelétricos, sem uma investigação detalhada dos lugares onde se pretende implantar. Os custos foram obtidos consultando-se diretamente os fabricantes de equipamentos e o mercado da praça de Ponta Grossa - PR. Os resultados mostraram que, para as microcentrais hidrelétricas, sempre que os custos constituírem o aspecto dominante, e para potências até $50 \mathrm{~kW}$, a opção por Bombas Funcionando como Turbinas (BFTs) deve ser considerada em lugar das turbinas hidráulicas.
\end{abstract}

PALAVRAS-CHAVE: microcentral, energia elétrica, bombas hidráulicas.

\section{COST OF PUMPS AS HYDRAULIC TURBINES FOR MICRO-SCALE HYDROPOWER}

SUMMARY: The implantation of micro hydroelectric power stations is one of the alternatives to supply energy to small and isolated communities, normally located in rural areas. The use of small load hydraulic potential is an alternative more feasible nowadays, not only because it lacks financial resources for big undertakings, but also, due to the small hydro power station hydraulic generation potential, which just a very small part has been taken advantage of. The objective of this study was to show a simple engineering methodology in order to estimate the electro-mechanic (generating set) equipment costs used in micro hydroelectric power plants, which can be applied on preliminary studies about new hydroelectric power stations to be built, without detailed investigation about the places where they should be implanted. Pumps As Turbines (PAT) have been studied. These equipment costs were obtained by consulting directly the manufacturers, and also the Ponta Grossa - PR city, Brazil, market. The results have shown that, concerning the micro hydroelectric power plants, whenever the costs constitute the major aspect and always considering units until $50 \mathrm{~kW}$ power, the option to PAT must be considered instead of hydraulic turbines.

KEYWORDS: micro hydroelectric power, electrical energy, hydraulic pumps.

\footnotetext{
${ }_{1}^{1}$ Prof. Associado, Departamento de Engenharia Civil, UEPG-PR, Ponta Grossa - PR, (0xx42) 222 7456, e-mail: crbalarim@uepg.br

${ }^{2}$ Prof. Titular, Departamento de Engenharia Rural, FCA/UNESP, Botucatu - SP, e-mail: targa@fca.unesp.br

${ }^{3}$ Prof. Adjunto, Departamento de Informática, UEPG-PR, Ponta Grossa - PR, e-mail: jvirgens@uepg.br

${ }^{4}$ Prof. Adjunto, Departamento de Engenharia Civil, UEPG-PR, Ponta Grossa - PR, e-mail: agafilho@uepg.br

${ }^{5}$ Prof. Assistente, Departamento de Engenharia Civil, UEPG-PR, Ponta Grossa - PR, e-mail: wiecheteck@interponta.com.br Recebido pelo Conselho Editorial em: 4-12-2001
}

Aprovado pelo Conselho Editorial em: 2-2-2004 


\section{INTRODUÇÃO}

Este trabalho foi conduzido com o objetivo de obter uma equação para a estimativa inicial do custo dos equipamentos eletromecânicos, utilizando Bombas Funcionando como Turbinas (BFTs), que, juntamente com os custos das demais estruturas permitirão, de forma aproximada, estimar o volume de recursos necessários à implantação de uma microcentral hidrelétrica (aproveitamento hidrelétrico com potência de até $100 \mathrm{~kW}$ ).

MACINTYRE (1983) e SOUZA et al. (1983) afirmam que o funcionamento de qualquer usina hidrelétrica depende fundamentalmente dos equipamentos eletromecânicos que compreendem os grupos geradores (turbinas, geradores elétricos e reguladores de velocidade).

As turbinas hidráulicas convencionais empregadas em usinas hidrelétricas de pequeno porte podem ser do tipo Francis, Pelton e Axiais (hélices, Kaplan, Tubulares, Bulbo), conforme MACINTYRE (1983).

Turbinas hidráulicas não convencionais têm sido desenvolvidas principalmente para uso em aproveitamentos de pequeno porte. No Brasil, essas turbinas têm sido pesquisadas e fabricadas para atender potências inferiores a $100 \mathrm{~kW}$, ou seja, dentro dos limites das microcentrais hidrelétricas. Estas máquinas são simples, com os objetivos de ter um custo reduzido e um rendimento aceitável. Dentre os seus tipos, destacam-se as turbinas Michell-Banki e Turgo, e as Bombas Funcionando como Turbinas (BFTs) (VIANA, 1997).

A idéia de utilizar bombas como turbinas para geração de energia elétrica ou acionamento mecânico não é recente. Seu uso é justificado por serem menos complexas, isto é, mais fáceis de instalar, manter e operar, e por serem produzidas em grande escala, sendo facilmente encontradas no mercado, em diversos modelos. O comportamento de bombas hidráulicas sob condições especiais de operação é bem conhecido desde a década de 30 (STEPANOFF, 1967).

As bombas operando em reverso como turbinas, para gerar energia em pequenos recursos hidroenergéticos, têm sido utilizadas em países como EUA, Alemanha e França. Bombas essas desde as centrífugas de vários estágios, de um estágio, mistas, até as axiais, substituindo as turbinas Pelton, Francis e hélices.

Para que a bomba funcione como turbina, o sentido do fluxo de escoamento da água se inverterá e, conseqüentemente, haverá a inversão no sentido de rotação.

Na Figura 1, apresenta-se o funcionamento de uma bomba centrífuga como bomba e como turbina. No caso do funcionamento como bomba, mostrado na Figura 1a, o líquido entra na sucção a baixa pressão, transforma energia de velocidade em pressão através do rotor, saindo pela descarga. No funcionamento como turbina, mostrado na Figura 1b, o líquido entra com energia de pressão, aciona o rotor em reverso e sai com baixa pressão.

No Brasil, em 1988, surgiu o projeto BFT (Bombas Funcionando com Turbinas), coordenado pela Escola Federal de Engenharia de Itajubá (EFEI), com o objetivo de desenvolver pesquisas e testes com vários tipos de bombas e apresentá-las como uma alternativa simples para a eletrificação do meio rural. Desse projeto, resultaram as publicações de SANTOS \& MARTINEZ (198-), SOUZA \& VIANA (198-), VIANA et al. (1986) e a proposta de duas metodologias para seleção de equipamentos (SOUZA \& VIANA, 198-), que apresentam de forma detalhada o comportamento e os aspectos operacionais da utilização em modo reverso de bombas centrífugas.

Na Figura 2, apresenta-se o esboço de uma instalação de Bomba Funcionando como Turbina. Os acessórios necessários para o funcionamento adequado dessa instalação são: uma curva de $90^{\circ}$ com diâmetro igual ao diâmetro da boca de entrada da bomba, destinada a operar a turbina, e um tubo de sucção cônico divergente com ângulo central de $12^{\circ}$, tendo o diâmetro menor igual ao diâmetro da 
curva de $90^{\circ}$ e com comprimento suficiente para penetrar $30 \mathrm{~cm}$ a partir do nível de água do canal de fuga.

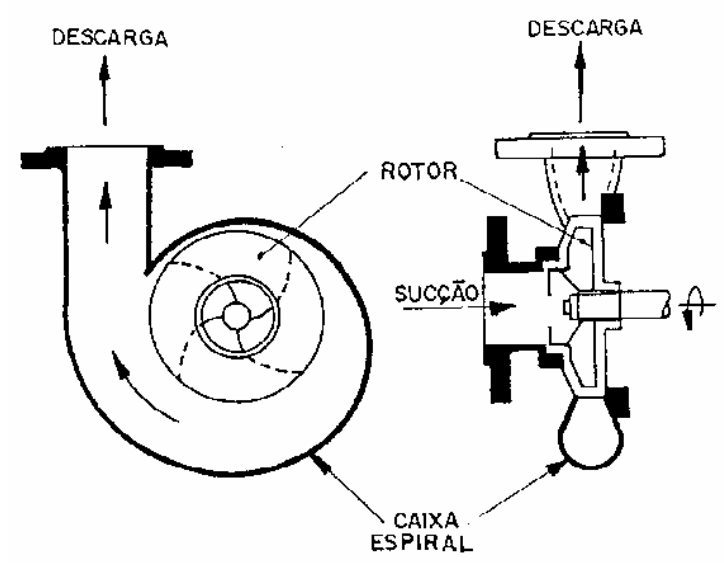

Bomba centrífuga funcionando como bomba.

(a)

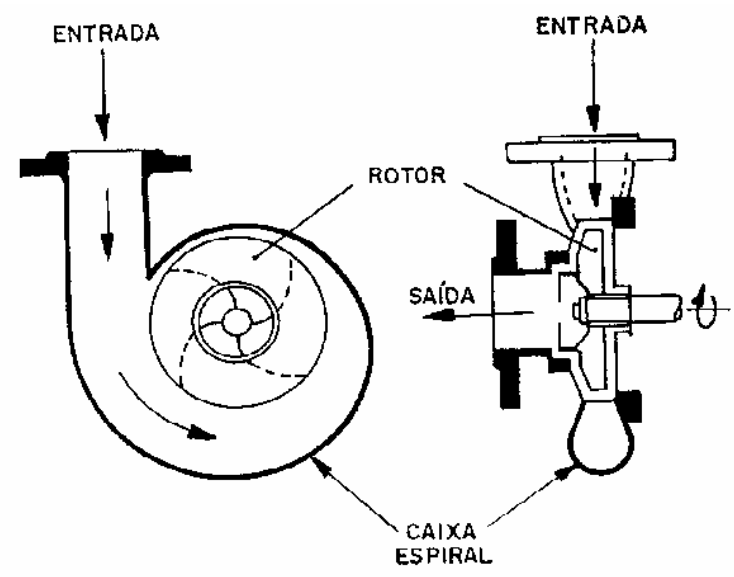

Bomba centrífuga funcionando como turbina.

(b)

FIGURA 1. Bomba centrífuga funcionando como bomba e como turbina. Fonte: VIANA, 1987.

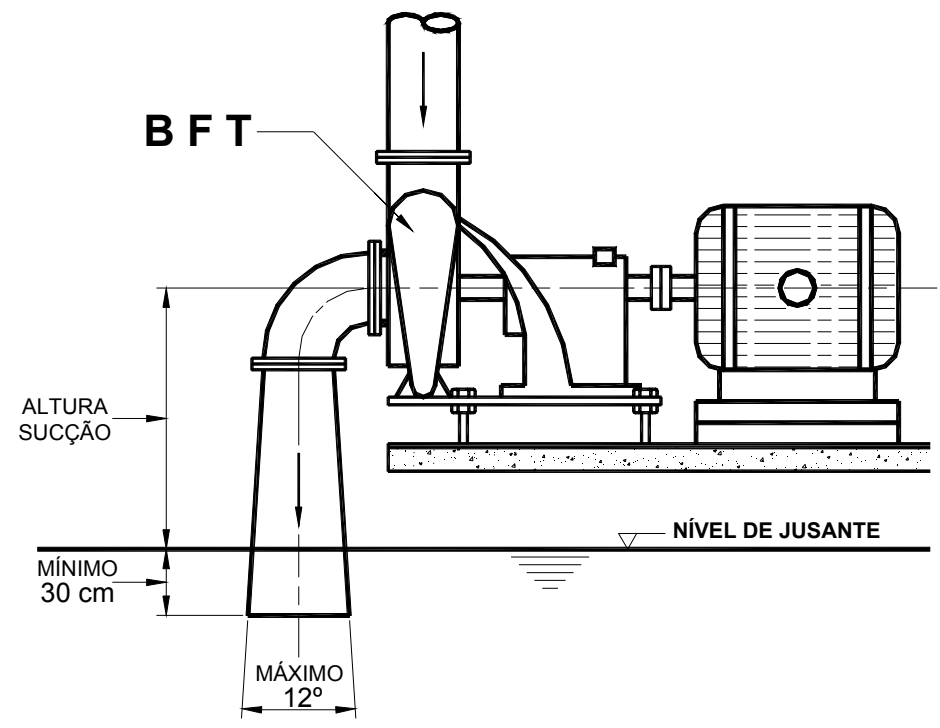

FIGURA 2. Bomba funcionando como turbina. Fonte: SOUZA et al. (1983)

Pesquisas realizadas por VIANA (1987), com bombas centrífugas, mostram a viabilidade de utilizar Bombas Funcionando como Turbinas (BFTs), mas recomendam utilizá-las com potências abaixo de $50 \mathrm{~kW}$.

Na Figura 3, indica-se a disponibilidade no mercado de bombas centrífugas passíveis de funcionamento como turbinas.

A escolha de uma turbina é feita em função das condições de vazão e de queda existentes no local. Segundo VIANA (1997), o campo de aplicação das BFTs é o indicado na Tabela 1. 


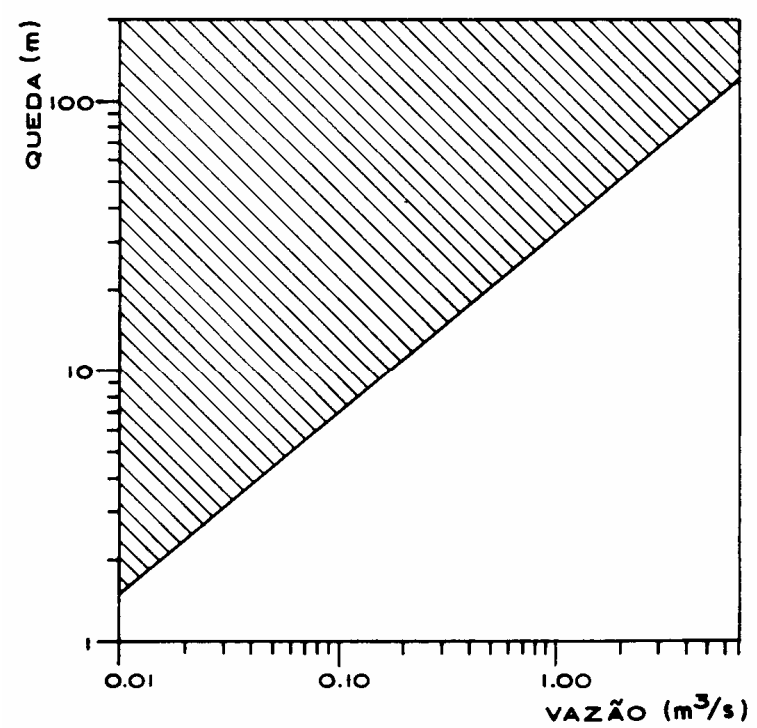

FIGURA 3. Região preferencial para aplicação de bombas centrífugas utilizadas em modo reverso. (fonte: BELUCO, 1994)

TABELA 1. Campo de aplicação das BFTs.

\begin{tabular}{cccc}
\hline Tipo da Turbina & Faixa de quedas $(\mathrm{m})$ & Vazões $\left(\mathrm{m}^{3} \mathrm{~s}^{-1}\right)$ & Faixa de Potências $(\mathrm{kW})$ \\
\hline BFTs & $6-100$ & $0,02 \mathrm{a} 0,25$ & $<50$ \\
\hline
\end{tabular}

As estimativas de custos das estruturas e equipamentos dos pequenos aproveitamentos têm sido pouco contempladas na literatura especializada. Os diversos estudos desenvolvidos nessa área dedicaram-se principalmente a centrais com capacidade instalada acima de $500 \mathrm{~kW}$, existindo pouco material para usinas com potências abaixo desse valor.

Para o caso de micro-usinas, SANT'ANA (1988) utilizou informações da publicação "Sistemática de cálculo para determinação do custo do $\mathrm{kW}$ instalado dos componentes hidromecânicos e elétricos de microusinas hidrelétricas (GUIMARÃES \& ROCHA, 1983).

Para esse tipo de central, ELETROBRÁS \& DNAEE (1985) também sugerem para a estimativa de custo de equipamentos uma tabela, na qual o valor é fornecido como uma função da queda (de 5 a $30 \mathrm{~m})$ e da potência (de 5 a $100 \mathrm{~kW}$ ).

BELUCO (1994) determinou o custo de turbinas hidráulicas tradicionais (Francis e Pelton), de turbinas Michell-Banki e de BFTs, utilizadas em microcentrais hidrelétricas.

\section{MATERIAL E MÉTODOS}

As bombas utilizadas para operarem em reverso como turbinas são as bombas de fluxo, ou seja, bombas centrífugas, mistas e axiais. Foram estudadas as bombas centrífugas operando em reverso com potências abaixo de $50 \mathrm{~kW}$.

Para a obtenção de dados de custo dos equipamentos eletromecânicos da casa de máquinas, os quais geralmente representam uma grande parcela do custo total da obra, principalmente nas pequenas usinas, foram consultados diretamente os fabricantes de equipamentos, o trabalho de BELUCO (1994) e o mercado da praça de Ponta Grossa - PR. Na ocasião, março de 1998, o dólar americano estava cotado em R\$ 1,13 . 
$\mathrm{Na}$ avaliação do custo dos equipamentos eletromecânicos, estão incluídos os seguintes componentes: conjunto turbina-gerador síncrono, assim como os equipamentos complementares (regulador de carga, válvula borboleta, curva de sucção, tubo de sucção, grade e quadro de comando).

A equação de custo, estimada em reais (R\$), foi determinada por meio de um modelo matemático ajustado com os dados levantados, utilizando o procedimento estatístico de análise de regressão (HOFFMANN \& VIEIRA, 1977). Foram avaliados os seguintes modelos:

- Modelo linear: $\mathrm{Y}=\mathrm{A}+\mathrm{B} \mathrm{X}$;

- Modelo potencial: $\mathrm{Y}=\mathrm{A} \mathrm{X}^{\mathrm{B}}$, que, por anamorfose, pode ser linearizado na forma $\ln (\mathrm{Y})=\ln (\mathrm{A})+\mathrm{B} \ln (\mathrm{X})$, por meio de transformação bilogarítmica das variáveis dependente $\mathrm{Y}$ e independente $\mathrm{X}$;

- Modelo exponencial: $\mathrm{Y}=\mathrm{A} \mathrm{B}^{\mathrm{X}}$, que, por anamorfose, pode ser linearizado na forma $\ln (\mathrm{Y})=\ln (\mathrm{A})+\mathrm{X} \ln (\mathrm{B})$, por meio de transformação monologarítmica da variável dependente Y.

Após as respectivas transformações logarítmicas nos dados, em que a variável Y representa o custo dos equipamentos eletromecânicos utilizando Bombas Funcionando como Turbinas $\left(\mathrm{C}_{\mathrm{EEBFT}}\right)$ e $\mathrm{X}$ a potência instalada $(\mathrm{P})$, foram estimados, pelo método dos mínimos quadrados, os coeficientes A e B dos modelos avaliados. Considerou-se como modelo mais adequado aquele que obteve o coeficiente de determinação $\left(\mathrm{r}^{2}\right)$ mais próximo da unidade. O ajuste dos modelos estatísticos foi realizado utilizandose do programa "STATISTICA for Windows", versão 4.3 da "StatSoft, Inc".

\section{RESULTADOS E DISCUSSÃO}

Realizada a análise de dados da planilha de custo dos equipamentos eletromecânicos utilizando Bombas Funcionando como Turbinas (BFTs), por meio da regressão aplicada aos modelos linearizados, verificou-se que o modelo exponencial linearizado foi o que apresentou o $\mathrm{r}^{2}$ mais próximo da unidade.

Os valores para as estimativas dos parâmetros, com os respectivos desvios-padrão e coeficiente de determinação, são mostrados na Tabela 2 .

TABELA 2. Estimativas dos parâmetros do modelo exponencial linearizado, com os respectivos desvios-padrão das estimativas dos parâmetros e coeficiente de determinação, para cálculo estimado do custo dos equipamentos eletromecânicos utilizando Bombas Funcionando como Turbinas (BFTs).

\begin{tabular}{cccc}
\hline Coeficientes & Estimativas dos Parâmetros & $\begin{array}{c}\text { Desvios-Padrão das } \\
\text { Estimativas dos Parâmetros }\end{array}$ & $\begin{array}{c}\text { Coeficiente de } \\
\text { Determinação }\left(\mathrm{r}^{2}\right)(\%)\end{array}$ \\
\hline $\mathrm{A}$ & 7,799766 & 0,029454 & \\
$\mathrm{~B}$ & 0,024897 & 0,001147 & 97,92 \\
\hline
\end{tabular}

Dessa forma, o modelo exponencial linearizado será dado por:

$\ln (Y)=7,799766+X \quad 0,024897$

Aplicando a base $e$ para reversão ao modelo exponencial a eq.(1) fica:

$\mathrm{Y}=2.440,03091,0252^{\mathrm{X}}$ 
Como $\mathrm{Y}=\mathrm{C}_{\mathrm{EEBFT}}$ e $\mathrm{X}=\mathrm{P}$, a estimativa do custo dos equipamentos eletromecânicos utilizando Bombas Funcionando como Turbinas (BFTs) será dada pela eq.(3), ou seja:

$$
\mathrm{C}_{\text {EEBFT }}=2.440,03091,0252^{\mathrm{P}}
$$

em que,

$\mathrm{C}_{\mathrm{EEBFT}}$ - custo dos equipamentos eletromecânicos utilizando BFTs, $\mathrm{R} \$, \mathrm{e}$

$\mathrm{P}$ - potência instalada, $\mathrm{kW}$.

Com a utilização da eq.(3), gerou-se a curva da Figura 4, que fornece as estimativas de custo para os equipamentos eletromecânicos utilizando Bombas Funcionando como Turbinas (BFTs) para as potências estudadas.

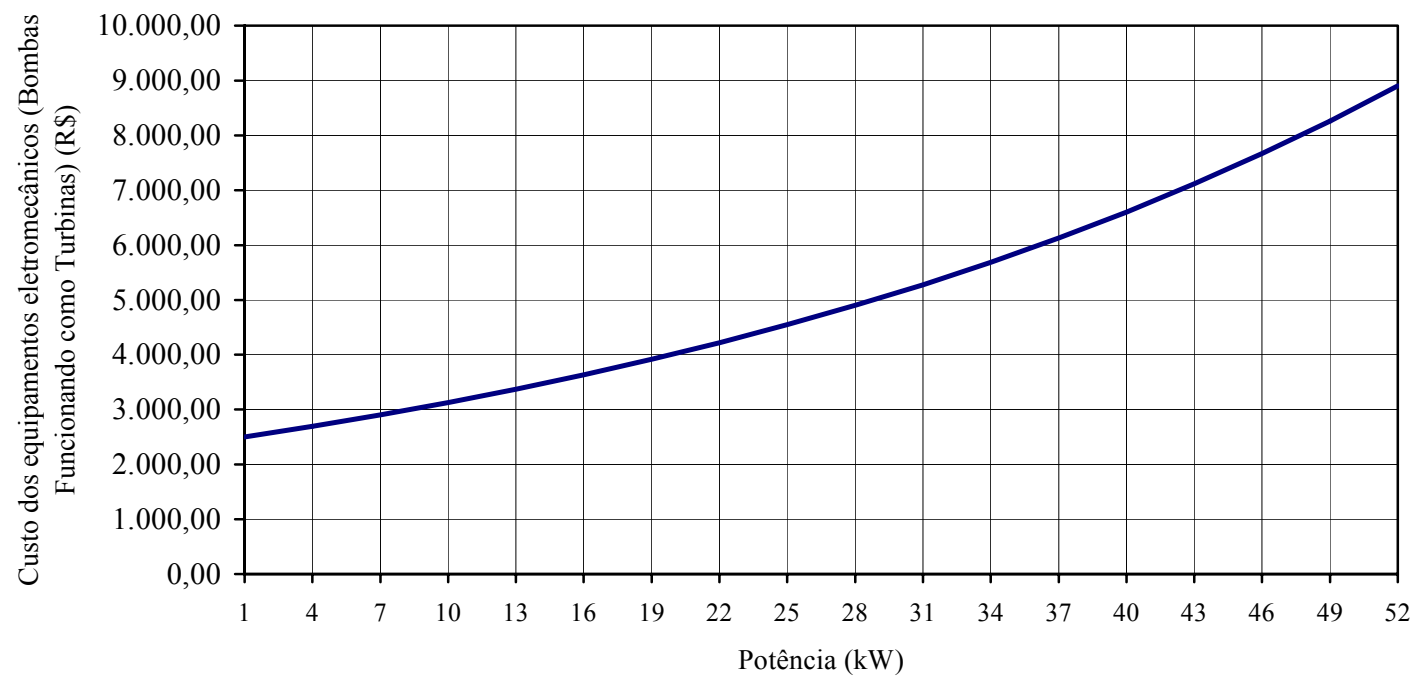

FIGURA 4. Custo dos equipamentos eletromecânicos utilizando (BFTs) em função da potência.

O resultado mostrou que o custo dos equipamentos eletromecânicos por $\mathrm{kW}$ instalado diminui com o aumento da potência quando se utilizam Bombas Funcionando como Turbinas (BFTs).

\section{CONCLUSÕES}

As Bombas Funcionando como Turbinas (BFTs) têm sido uma solução simples em aproveitamentos de pequeno porte em diversos países, além do que, até a faixa de potências em que podem ser empregadas $(50 \mathrm{~kW})$, as mesmas são encontradas facilmente no mercado.

O emprego de bombas centrífugas para esse tipo de aproveitamento deve ser considerado como uma alternativa atrativa, visto que esses equipamentos, também, apresentam bom desempenho e exigem poucas modificações para sua adoção em funcionamento reverso.

$\mathrm{O}$ trabalho mostrou que esse tipo de equipamento apresenta custo por $\mathrm{kW}$ instalado variando de $\mathrm{R} \$ 2.500,00$ (US\$ 2.212,00) para potência de $1 \mathrm{~kW}$ até $\mathrm{R} \$ 170,00$ (US\$ 150,00) para $50 \mathrm{~kW}$, e que, na faixa inferior de potências das microcentrais hidrelétricas (até $25 \mathrm{~kW}$ ), esse custo por $\mathrm{kW}$ instalado decresce continuamente com o aumento da potência, tendendo a estabilizar-se em R $\$ 170,00$ na faixa superior (de 25 a $50 \mathrm{~kW}$ ).

O resultado obtido, embora não seja completamente conclusivo, poderá ser generalizado e utilizado de modo a racionalizar e simplificar os estudos de avaliação preliminar para implantação de microaproveitamentos dentro da faixa de potência possível para utilização desse tipo de equipamento. 


\section{REFERÊNCIAS BIBLIOGRÁFICAS}

BELUCO, A. Viabilidade de microcentrais hidrelétricas baseadas no emprego de equipamentos de mercado. 1994. 170 f. Dissertação (Mestrado em Energia) - Escola de Engenharia, Universidade Federal do Rio Grande do Sul, Porto Alegre, 1994.

ELETROBRÁS. DEPARTAMENTO NACIONAL DE ÁGUAS E ENERGIA ELÉTRICA. Manual de microcentrais hidrelétricas. Rio de Janeiro, 1985. 584 p.

GUIMARÃES, L.B.; ROCHA, J.C.A. Sistemática de cálculo para a determinação do $\mathrm{kW}$ instalado dos componentes hidromecânicos e elétricos de microusinas hidrelétricas. Curitiba: Secretaria de Estado de Planejamento, Coordenadoria de Estudos e Projetos, 1983. 58 p.

HOFFMANN, R.; VIEIRA, S. Análise de regressão: uma introdução a econometria. São Paulo: Editora HUCITEC, 1977. 379 p.

MACINTYRE, A.J. Máquinas motrizes hidráulicas. Rio de Janeiro: Guanabara Dois, 1983. 654 p.

SANT'ANA, R.F. Aproveitamentos hidrelétricos de pequeno porte. Avaliação de custos e benefícios de pequenas centrais hidrelétricas. Brasília: DNAEE/COPEL,1988. 247 p.

SANTOS, A.H.M.; MARTINEZ, C.B. Tecnologia apropriada para microcentrais hidráulicas. Itajubá: Escola Federal de Engenharia, 198-. 14 p. (Publicação Interna)

SOUZA, Z.; FUCHS, R.D.; SANTOS, A.H.M. Centrais hidro e termelétricas. São Paulo: Edgard Blucher, 1983. 244 p.

SOUZA, Z.; VIANA, A.N.C. Bombas funcionando como turbinas. Itajubá: Escola Federal de Engenharia, 198-. 19 p. (Publicação interna do Laboratório Hidromecânico para Pequenas Centrais Hidrelétricas)

STEPANOFF, A.J. Centrifugal and axial flow pumps. 2. ed. New York: John Wiley and Sons, 1967. $462 \mathrm{p}$.

VIANA, A.N.C. Comportamento de bombas centrifugas funcionando como turbinas hidráulicas. 1987. 125 f. Dissertação (Mestrado em Engenharia Mecânica) - Escola Federal de Engenharia de Itajubá, Itajubá, 1987.

VIANA, A.N.C. Pequenas centrais hidrelétricas no meio rural. In: SERAPHIM, O.J. (Ed.) Tecnologia e aplicação racional de energia elétrica e de fontes renováveis. Campina Grande: SBEA/UFPB, 1997. p.135-64.

VIANA, A.N.C.; TIAGO, F.; LEITE, F. Bombas centrífugas funcionando como turbinas, para microcentrais. Mundo Elétrico, São Paulo, n.320, p.52-5, 1986. 